2015-12

Simplest relationship between local field potential and intracellular signals in layered neural tissue.

Chizhov, AV

http://hdl.handle.net/10026.1/4834

10.1103/PhysRevE.92.062704

Phys Rev E Stat Nonlin Soft Matter Phys

All content in PEARL is protected by copyright law. Author manuscripts are made available in accordance with publisher policies. Please cite only the published version using the details provided on the item record or document. In the absence of an open licence (e.g. Creative Commons), permissions for further reuse of content should be sought from the publisher or author. 


\section{Simplest relationship between local field potential and intracellular signals in layered neural tissue}

Article in Physical Review E · December 2015

Impact Factor: 2.29 · DOI: 10.1103/PhysRevE.92.062704

READS

60

4 authors, including:

\section{Anton Chizhov}

Ioffe Physical Technical Institute

42 PUBLICATIONS 123 CITATIONS

SEE PROFILE 


\title{
Simplest relationship between local field potential and intracellular signals in layered neural tissue
}

\author{
Anton V. Chizhov,,${ }^{1,2, *}$ Alberto Sanchez-Aguilera, ${ }^{3}$ Serafim Rodrigues,${ }^{4}$ and Liset Menendez de la Prida ${ }^{3}$ \\ ${ }^{1}$ Ioffe Institute, RAS, Politekhnicheskaya str., 26, 194021, St.-Petersburg, Russia \\ ${ }^{2}$ Sechenov Institute of Evolutionary Physiology and Biochemistry of RAS, Torez pr. 44, 194223, St.-Petersburg, Russia \\ ${ }^{3}$ Instituto Cajal-CSIC, Ave. Doctor Arce, 37, Madrid 28002, Spain \\ ${ }^{4}$ Centre for Robotics and Neural Systems, School of Computing and Mathematics, Plymouth University, Drake Circus, \\ Plymouth, Devon PL4 8AA, United Kingdom
}

(Received 17 June 2015; revised manuscript received 9 October 2015; published 7 December 2015)

\begin{abstract}
The relationship between the extracellularly measured electric field potential resulting from synaptic activity in an ensemble of neurons and intracellular signals in these neurons is an important but still open question. Based on a model neuron with a cylindrical dendrite and lumped soma, we derive a formula that substantiates a proportionality between the local field potential and the total somatic transmembrane current that emerges from the difference between the somatic and dendritic membrane potentials. The formula is tested by intraand extracellular recordings of evoked synaptic responses in hippocampal slices. Additionally, the contribution of different membrane currents to the field potential is demonstrated in a two-population mean-field model. Our formalism, which allows for a simple estimation of unknown dendritic currents directly from somatic measurements, provides an interpretation of the local field potential in terms of intracellularly measurable synaptic signals. It is also applicable to the study of cortical activity using two-compartment neuronal population models.
\end{abstract}

DOI: 10.1103/PhysRevE.92.062704

PACS number(s): 87.18.Sn, 87.19.1d, 87.19.1j

\section{INTRODUCTION}

Extracellular potentials are commonly used in neurophysiology. However, an interpretation of these signals in terms of intracellular parameters of neuronal activity is unclear [1-5]. In particular, even in the case of synchronous neuronal epileptiform events the relationship between simultaneously registered extracellular and intracellular signals is nontrivial [6]. Assuming a homogeneous and isotropic extracellular space (but see Ref. [7]), an extracellular potential is commonly described by the Poisson equation with macroscopically averaged transmembrane currents as distributed sources [8,9] or by the Laplace equation with the transmembrane currents distributed along all membrane surfaces [10-15]. If neuronal activity is modeled by multiple compartment neurons, then calculation of the local field potential (LFP) with the Poisson equation is straightforward. However, in most cases researchers only have access to measure signals at the somatic compartment, making estimation of the contribution of single neurons to LFP challenging because their dendritic currents cannot be directly estimated. Moreover, biophysically detailed mean-field models also deals with somatic signals of neuronal activity exclusively, and the question of how to estimate the LFP from these somatic signals is unclear [16-18]. Instead of Poisson equation-based models, simple formulas linking intracellular parameters of neuronal activity to extracellular field potential have been proposed [19-24] coming from phenomenological considerations that are still debated [22,25]. Some of such simple phenomenological models include those that relate the LFP to: (a) the somatic membrane voltage [26,27], (b) the sum of absolute values of the excitatory and inhibitory synaptic currents [20,28], (c) the sum of synaptic and intrinsic currents of slow voltage-gated ionic channels [21], or (d) to the difference of dendritic and somatic

*anton.chizhov@mail.ioffe.ru membrane voltages [23]. Is there any possibility to analytically model extracellular field potentials resulting from dendritic synaptic activity using simple models of neurons?

In the present work, we derive an approximation of LFP that is compatible with two-compartment neuron-based population models and relates the LFP to somatic intracellular recordings that account for dendritic contribution. To that purpose, first the Poisson equation is applied to a distributed neuron (DN) consisting of a cylindrical dendrite of a given length stemming from the soma (Sec. II A), which gives a formula of LFP as a function of membrane potentials at the soma and dendrite (Sec. II B). Then, in order to apply the LFP formula in the framework of population modeling approaches, a twocompartment neuron (TCN) model is constructed, based only on somatic recordings in voltage- and current-clamp mode. Joint solution of two boundary problems each corresponding to one of these modes with linear approximation of the potential distribution along the dendrite constitutes the TCN (Sec. II C). Such a derivation, which was initially proposed in Ref. [29] for a particular case, is now generalized in the present work. The TCN model provides an estimation of the synaptic current at dendrite given the resulting current measured in the voltageclamp mode at the soma. In Sec. III, the derived formula for the LFP is verified with patch-clamp and extracellular recordings in hippocampal slices. The proposed TCN and LFP models are recommended for an implementation into a population firing-rate-type model proposed in Ref. [30] (Sec. IV) or a conductance-based refractory density model proposed in Ref. [31]. This model is suitable to examine the relationship between intracellular and LFP synaptic signals in layered neural tissue like the hippocampus or the neocortex.

\section{MATHEMATICAL MODEL OF LFP}

In order to construct a model for an extracellular potential measured on layered neural structures, we make the following assumptions: (1) the neuronal populations are homogeneously 


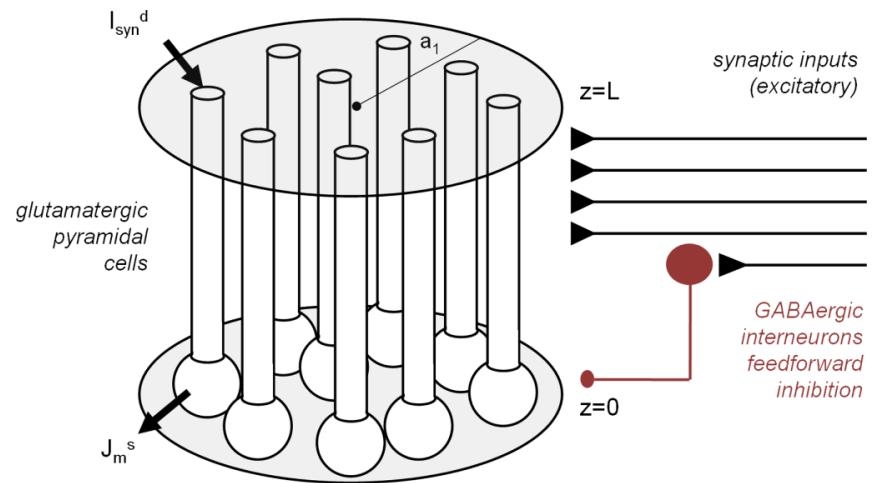

FIG. 1. (Color online) Schematic of the layered hippocampal structure under consideration and model details. Glutamatergic pyramidal cells are modeled as a passive cylindrical dendrite of length $L$ lumped to a soma at one extreme. Synaptic currents impinging at the dentrites $\left(I_{\text {syn }}^{d}\right)$ at $z=L$ flow into the $\left(J_{m}^{s}\right)$. The extracellular field potential is calculated along the $z$ axis of a conductive cylinder of radius $a_{1}$, with $z=0$ at the soma. Activation of the excitatory synaptic input pathway lead to dendritic synaptic inward currents impinging on aligned pyramidal cells at a distance from the soma $(L)$, as well as outward perisomatic synaptic inhibition resulting from feedforward activation of local circuit GABAergic interneurons.

distributed in the space; (2) only one-type (pyramidal) cells influence the extracellular potential; (3) the complexities of neuronal dendritic trees can be neglected and single equivalent dendrites are aligned in vertical direction; and (4) the extracellular potential changes are negligible for transmembrane potential calculations, i.e., $V=V_{\text {intra }}$. Note that only synaptic activities will be included, i.e., no active ionic currents are considered and compartments are passive. However, we introduce later a contribution of spikes in the LFP model (Sec. IV).

\section{A. Distributed neuron model (DN) of a single neuron}

We model a neuron as a passive cylindrical dendrite with the soma at one extreme, i.e., as a boundary condition. Therefore the neuron is considered a passive cable aligned along the vertical coordinate $z$, with soma at $z=0$ and the end of the dendrite at $z=L$ (Fig. 1). Synaptic currents input into the soma, $I_{\text {syn }}^{s}$, and at the end of the dendrite, $I_{\text {syn }}^{d}$. The cable equation for the membrane potential $V(t, z)$ along the distributed model neuron is

$$
\frac{1}{2 \pi a} \frac{\partial}{\partial z}\left(\frac{1}{r_{i}} \frac{\partial V}{\partial z}\right)=j_{m}(z, t)
$$

where $a$ is the radius of the dendrite and $r_{i}$ is the specific intracellular resistivity. The transmembrane current $j_{m}(t, z)$ is a sum of a leakage and capacity terms, i.e.,

$$
j_{m}(z, t)=C \frac{\partial V}{\partial t}+g_{L} V
$$

where $g_{L}$ is the specific leak conductance and $C$ is the specific capacitance. The voltage $V(z, t)$ is assumed to be measured from the resting level.
Under current clamp mode boundary conditions at the soma take the form:

$$
\left.\frac{1}{r_{i}} \frac{\partial V}{\partial z}\right|_{z=0}=\left(C \frac{d U}{d t}+g_{L} U\right) S^{\mathrm{soma}}-I_{\mathrm{syn}}^{s} \equiv J_{m}^{s}(t),
$$

where we use the notation $U(t) \equiv V(t, 0)$ for the measurable somatic voltage, $I_{\text {syn }}^{S}$ is the synaptic current impinging on the soma, $J_{m}^{s}$ is the total current flowing through somatic membrane, and $S^{\text {soma }}$ is the somatic membrane area. At the end of the dendrite, boundary conditions take the form:

$$
\left.\frac{1}{r_{i}} \frac{\partial V}{\partial z}\right|_{z=L}=I_{\text {syn }}^{d}
$$

\section{B. Extracellular field potential equations}

In order to get the next level approximation, we derive the LFP equation resulting from the DN model. Following Ref. [32] we consider a cylindrical layer of radius $a_{1}$ (Fig. 1), where neurons are homogeneously distributed with the density $p$ per area and their dendrites aligned along the radial coordinate $z$ from 0 to $L$. Our goal is to estimate the extracellular potential $\varphi(t, z)$ along the axis of symmetry.

Extracellular potential in a conductive medium is calculated as a superposition of current sources and described by the Poisson equation [8]:

$$
\nabla \cdot(\sigma \nabla \varphi)=-J
$$

where $J$ is the average of the inward currents per unit volume and $\sigma$ is the mean conductivity of extracellular medium, assumed to be a constant tensor. For a one-dimensional source function $J(t, z)$ distributed along the cylinder, the solution of (5) for the extracellular potential at $z=z_{0}$ is given by the convolution [32]:

$$
\varphi\left(t, z_{0}\right)=\int_{0}^{L} G\left(z_{0}, z\right) J(t, z) d z,
$$

with the kernel that reflects the potential at the axis of symmetry of a unit charge distributed on a disk with radius $a_{1}$ [33]: $G\left(z_{0}, z\right)=\left(\sqrt{\left(z_{0}-z\right)^{2}+a_{1}^{2}}-\left|z_{0}-z\right|\right) / 2 \sigma$. (Though the potential distribution off the $z$ axis is not planar for limited disk.) The potential within and near the thin layer $\left(L \ll a_{1}\right)$ can be approximated for $\left|z_{0}-z\right| \ll a_{1}$ as

$$
G\left(z_{0}, z\right) \approx\left(a_{1}-\left|z_{0}-z\right|\right) / 2 \sigma .
$$

For the system of distributed parallel dendrites (i.e., DN model) with boundary sources at $z=0$ and $z=L$ :

$$
\begin{aligned}
J(t, z)= & 2 \pi a p j_{m}(t, z)+p J_{m}^{s}(t) \delta(z-0) \\
& -p I_{\text {syn }}^{d}(t) \delta(z-L) .
\end{aligned}
$$

After substitution (7) and (8) into (6), the potential $\varphi$ becomes:

$$
\begin{aligned}
\varphi\left(t, z_{0}\right)= & \frac{p}{2 \sigma}\left[2 \pi a \int_{0}^{L} j_{m}(t, z)\left(a_{1}-\left|z_{0}-z\right|\right) d z\right. \\
& \left.+J_{m}^{s}(t)\left(a_{1}-z_{0}\right)-I_{\text {syn }}^{d}(t)\left(a_{1}-\left|z_{0}-L\right|\right)\right] .
\end{aligned}
$$

Now we note that the transmembrane current $j_{m}$ may be calculated from Eq. (1). Thus substituting Eq. (1), the first 
term in commas is rewritten and then integrated by parts with the boundary conditions defined in Eqs. (3) and (4), as follows:

$$
\begin{aligned}
2 \pi & a \int_{0}^{L} j_{m}(t, z)\left(a_{1}-\left|z_{0}-z\right|\right) d z \\
= & \int_{0}^{L} \frac{1}{r_{i}} \frac{\partial^{2} V}{\partial z^{2}}\left(a_{1}-\left|z_{0}-z\right|\right) d z \\
= & \left(a_{1}-\left|z_{0}-L\right|\right) I_{\text {syn }}^{d}(t)-\left(a_{1}-z_{0}\right) J_{m}^{s}(t) \\
& -\left[2 V\left(t, \min \left(z_{0}, L\right)\right)-V(t, 0)-V(t, L)\right] / r_{i} .
\end{aligned}
$$

Inserting this expression into $(9), \varphi(t, z)$ is obtained as

$$
\varphi\left(t, z_{0}\right)=\frac{p}{2 \sigma r_{i}}\left[-2 V\left(t, \min \left(z_{0}, L\right)\right)+V(t, 0)+V(t, L)\right] .
$$

Note that this simple formula is obtained because of the similarity of the Poisson and cable equations. Considering the position of somas $\left(z_{0}=0\right)$ and dendrites $\left(z_{0}=L\right)$, the somatic and dendritic extracellular potentials are

$$
\varphi(t, 0)=-\varphi(t, L)=\frac{p}{2 \sigma r_{i}}[V(t, L)-V(t, 0)] .
$$

Note that the extracellular potentials at the levels of somas and synapses are opposite in signs. As follows from (10), the distribution of $\varphi$ along the $z$ axis is a linear combination of the somatic and dendritic membrane potentials. This approximation is roughly consistent with depth multielectrode measurements of LFP in layered structures [34]. It reveals the main intracellular terms contributing in LFP (after zero term in one-compartment models), showing the consistency of the conventional representation of LFP by a dipole.

In order to meet the typical experimental constraints, we eliminate nonsomatic measures from the LFP estimation by applying a linear approximation of the membrane potential along the dendrite and rewriting formula (11) with the help of (3) as follows:

$$
\varphi(t, 0)=-\varphi(t, L)=\frac{p L}{2 \sigma} J_{m}^{s}(t) .
$$

Equations (11) or (12) constitute the model of LFP expressed as the difference of the measurable somatic transmembrane potentials or membrane current defined by (3) as a sum of capacitive, leak, and synaptic currents. Note that a singlecompartment consideration would imply the transcompartment current in the left-hand part of (3) to be 0 , thus the total transmembrane current $J_{m}^{s}$ in the right-hand part is always zero, too. Because $J_{m}^{s}$ is the major contributor to LFP according to Eq. (12), one-compartment models cannot be used to simulate LFP signals.

\section{Two-compartment neuron model (TCN)}

In order to apply the LFP formula (12) in the framework of population modeling approaches, at least a two-compartment consideration is required that copes for dendritic effects on somatic recordings. To this end, we now derive a TCN from the DN model, extending the derivation from Ref. [29] to the case of arbitrary dendritic length. In contrast to the conventional consideration [35], we base our model only on somatic variables that can be determined experimentally using patch recordings. Our purpose is to relate somatic currents recorded under conditions of fixed voltage (i.e., voltage-clamp) to voltage deflections recorded in current-clamp conditions. These two regimes correspond to two different boundary problems for the spatially distributed neuron described by Eqs. (1) and (2). The current-clamp (CC) case is set by the boundary conditions (3) and (4), whereas for the voltageclamp (VC) case the boundary condition (3) is substituted by $V(t, 0)=0$ (i.e., resting membrane potential) and the recorded current is given by the current flux through the soma, i.e.,

$$
I_{\mathrm{VC}}=\left.\frac{1}{r_{i}} \frac{\partial V}{\partial z}\right|_{z=0}+I_{\mathrm{syn}}^{s} .
$$

The two-compartment model equations are derived as a reduction of the equations for the two joint boundary problems [(1)-(4)] with the help of a linear approximation of the voltage distribution along the dendrite with the extreme values of $U$ at the soma and $U_{d}$ at the center of the dendrite, as proposed in Ref. [29]:

$$
V(t, z)=U(t)(1-2 z / L)+2 U_{d}(t) z / L .
$$

For the first VC boundary problem, integration of Eq. (1) along the dendrite with (14) and $U(t)=0$ gives

$$
L\left(C \frac{d U_{d}}{d t}+g_{L} U_{d}\right)=\frac{1}{2 \pi a}\left(I_{\mathrm{syn}}^{d}-\frac{U_{d}}{r_{i} L}\right) .
$$

For the VC problem we know the measured somatic current $I_{\mathrm{VC}}$, whereas the synaptic current at the distal part of the dendrite $I_{\text {syn }}^{d}$ is experimentally unknown. Linear approximation (14) applied to (13) gives $I_{\mathrm{VC}}-I_{\text {syn }}^{s}=2 U_{d} /\left(r_{i} L\right)$. With this expression we can eliminate $U_{d}$ from Eq. (15) and obtain

$$
I_{\text {syn }}^{d}=\left[\pi a L^{2} r_{i} C \frac{d}{d t}+1+\pi a L^{2} r_{i} g_{L}\right]\left(I_{\mathrm{VC}}-I_{\text {syn }}^{s}\right) \text {. }
$$

Introducing the characteristic length $\lambda=1 / \sqrt{2 \pi a g_{L} r_{i}}$ and the membrane time constant at rest $\tau_{m}^{0}=C / g_{L}$, we get

$$
I_{\text {syn }}^{d}=\left[\frac{L^{2}}{2 \lambda^{2}} \tau_{m}^{0} \frac{d}{d t}+1+\frac{L^{2}}{2 \lambda^{2}}\right]\left(I_{\mathrm{VC}}-I_{\text {syn }}^{s}\right) .
$$

Next we solve the boundary problem for the $\mathrm{CC}$ regime. The somatic voltage is now determined by the boundary condition (3), which for the linear approximation (14) reads as follows:

$$
\left(C \frac{d U}{d t}+g_{L} U\right) S^{\text {soma }}-I_{\text {syn }}^{s}=\frac{2}{r_{i}} \frac{U_{d}-U}{L} .
$$

Integration of (1) with (14) and boundary conditions (18) and (4) gives

$$
C \frac{d U_{d}}{d t}+g_{L} U_{d}=\frac{I_{\mathrm{syn}}^{d}}{\pi a L}-2 \frac{U_{d}-U}{\pi a L^{2} r_{i}} .
$$

The estimate of the synaptic current at the distal end of the dendrite $I_{\text {syn }}^{d}$, obtained from the somatic current $I_{\mathrm{VC}}$ by Eq. (17), is then substituted in the Eqs. (18) and (19) of the CC boundary problem. In spite of low precision of $I_{\text {syn }}^{d}$ estimation, seen in comparison with the original synaptic current in the distributed model, the joint solution of both direct and reverse problems provides much more precise approximation of the somatic voltage $U$. 
Finally, we rewrite (18), (19), and (17) as follows:

$$
\begin{gathered}
\tau_{m}^{0} \frac{d U}{d t}=-U+\frac{2 \gamma}{l}\left(U_{d}-U\right)+\frac{I_{\text {syn }}^{s}}{G^{s}}, \\
\tau_{m}^{0} \frac{d U_{d}}{d t}=-U_{d}-\frac{2}{l}\left(U_{d}-U\right)+\frac{I_{\text {syn }}^{d}}{\gamma G^{s}}, \\
I_{\text {syn }}^{d}=\left(\frac{l}{2} \tau_{m}^{0} \frac{d}{d t}+1+\frac{l}{2}\right)\left(I_{\mathrm{VC}}-I_{\text {syn }}^{s}\right),
\end{gathered}
$$

where $\gamma=S^{\text {dendr }} / S^{\text {soma }}, \quad l=L^{2} / \lambda^{2}, \quad G^{s}=g_{L} S^{\text {soma }}$, and $S^{\text {dendr }}=2 \pi a L$. Thus, Eqs. (20)-(22) govern the relationship between the somatic voltage $U$ and the synaptic currents measured at the soma, $I_{\text {syn }}^{s}$ and $I_{\mathrm{VC}}$, contributed by two synaptic inputs, one impinging on the soma $\left(I_{\text {syn }}^{s}\right)$ and another on the dendrite $\left(I_{\text {syn }}^{d}\right)$. In comparison to the Pinsky-Rinzel model [35], the proposed model provides an estimate of the somatodendritic coupling $g_{c}=\gamma g_{L} / l$ and the estimate for the dendritic synaptic current from its somatic estimation [Eq. (22)]. The input conductance at rest is given by $G_{\text {in }}=$ $G^{s}(3+2 \gamma) /(3+\gamma)$.

\section{EXPERIMENTAL RESULTS}

In this section we check our predictions experimentally by comparing extracellular field recordings with those estimated from somatic patch-clamp measurements. To this purpose we recorded intracellular and extracellular responses to electric stimulation in hippocampal slices known to have a layered structure (Fig. 1). The experimental microcircuit under consideration consists of aligned pyramidal cells that are directly activated by an input pathway targeting their dendrites at a distance from the somatic layer. A major feature of hippocampal and cortical circuits is perisomatic feedforward inhibition accompanying input pathway stimulation, which is caused by pathway activation of local GABAergic interneurons. Thus by stimulating extracellularly we will be able to evoke both, a dendritic excitatory synaptic current and a somatic inhibitory current in hippocampal cells.

In our experimental design, a stimulating bipolar electrode was located in the CA2 region of $400-\mu \mathrm{m}$-thick hippocampal slices, the recording patch-clamp pipette targeted CA1 pyramidal cells in stratum pyramidale and the field electrode was placed in the stratum pyramidale [Fig. 2(a)]. Note that this placement of the field electrode is equivalent to measure LFP, $\varphi(t, 0)$, as predicted by Eq. (12) or (13) at the somatic layer, i.e., the stratum pyramidale. Stimulation consisted of two pulses of $0.2 \mathrm{~ms}$ at a $50-\mathrm{ms}$ interval.

We adopted two experimental approaches to validate our model of LFP estimations. The first approach requires recordings in both voltage-clamp and current-clamp modes and uses dynamic computer control, whereas the second exploits the standard voltage-clamp mode solely. Both approaches encompass the most common recording modes available at electrophysiological labs nowadays.

\section{A. Experiment 1}

First, the somatic membrane potential $U(t)$ was measured in the current-clamp mode. Next, the compound synaptic current resulting from dendritic activation and feedforward perisomatic inhibition was measured in the soma under VC mode at different holding voltage levels. From these recordings the excitatory and inhibitory conductances $g_{E^{\prime} E}$ and $g_{I E}$ were estimated by the method from Ref. [36] using standard values for reversal potentials $V_{E}$ and $V_{I}$, as well as the resting voltage $V_{\text {rest }}$ and the input conductance at rest $G_{\text {in }}$. Finally, in order to estimate the compound somatic transmembrane current $\left[J_{m}^{s}(t)\right.$ in Eq. (12)], we recorded its nonsynaptic components, the somatic capacity and leak current by setting the initially recorded voltage response in $\mathrm{CC}$ mode as a profile of holding voltage in VC mode, i.e., $I_{C}^{S}+I_{L}^{S} \approx I_{\text {at }} V_{h}=U(t)$ (see the Appendix A). This was done by dynamic computer control of the amplifier (dynamic voltage-clamp). The total somatic transmembrane current is $J_{m}^{s}(t)=I_{C}^{s}+I_{L}^{s}+I_{\text {syn }}^{s}$, according to its definition (3). According to the circuit structure (Fig. 1), activation of the synaptic input pathway lead to dendritic synaptic currents $I_{\text {syn, } E^{\prime} E}^{d}$, as well as somatic synaptic inhibition $I_{\text {syn, } I E}^{S}$ resulting from feedforward activation of local circuit GABAergic interneurons. Thus we imply that $I_{\text {syn }}^{d}=$ $I_{\text {syn, } E^{\prime} E}^{d}$ and $I_{\text {syn }}^{s}=I_{\text {syn, } I E}^{s}$, and hence $I_{\mathrm{VC}}-I_{\text {syn }}^{s}=I_{\text {syn, } E^{\prime} E}^{s}$ in Eq. (22) used to estimate $I_{\mathrm{syn}, E^{\prime} E}^{d}$. This dendritic source $I_{\mathrm{syn}, E^{\prime} E}^{d}$ affects LFP through the capacitive and leak somatic currents. The somatically measured excitatory and inhibitory synaptic currents at $V_{\text {rest }}$ were estimated as $I_{\text {syn, } E^{\prime} E}=g_{E^{\prime} E}\left[U(t)-V_{E}\right]$ and $I_{\text {syn, } I E}^{s}=g_{I E}\left[U(t)-V_{I}\right]$. The formula (12) for LFP at the stratum pyramidale is then written as

$$
\varphi(t, 0)=\frac{p L}{2 \sigma}\left[I_{\text {at } V_{h}=U(t)}-I_{\text {syn }, I E}^{s}\right] .
$$

The values of parameters for estimations (11) and (12) are taken from the literature: the pyramidal cell density $p=8 \times$ $10^{4} \mathrm{~cm}^{-2}$ [37]; the bulk conductivity of extracellular medium along the $z$ axis $\sigma$ is estimated as the conductivity of interstitial fluid, $0.004 \mathrm{~S} / \mathrm{cm}$ [8], multiplied by the factor 0.2 derived in Ref. [38] from the consideration of extracellular space as a hexagonal array of cylinders; the specific intracellular resistivity $r_{i}$ is estimated by the intracellular longitudinal resistance $500 \Omega \mathrm{cm}$ [8] and the mean dendrite diameter $3 \mu \mathrm{m}$ to obtain $7 \times 10^{9} \Omega / \mathrm{cm}$; and, finally, the distance from soma to excitatory synapses, $L$, is taken as $200 \mu \mathrm{m}$ [39]. These values result in factors $p /\left(2 \sigma r_{i}\right)=0.007$ for $(11)$ and $p L /(2 \sigma)=1 M \Omega$ for (12) and (23).

Data are presented in Figs. 2(b) and 2(c) for two cells of different slices. The top plots represent the recorded postsynaptic potentials $U(t)$ (red traces) recorded at soma in $\mathrm{CC}$ mode. Below are the current components as estimated directly from somatic recordings: [Fig. 2(a)] a dendritically originated excitatory current $I_{\text {syn, } E^{\prime} E}^{s}$ (green trace), [Fig. 2(b)] the perisomatic inhibitory current $I_{\text {syn, } I E}^{s}$ (blue trace), and [Fig. 2(d)] the somatic capacity and leak current $I_{\text {at }} V_{h}=U(t)$ (light orange trace), recorded in dynamic VC mode.

The resulting current $J_{m}^{s}(t) \approx I_{\text {at } V_{h}=U(t)}-I_{\text {syn, } I E}^{s}$ allowed us to estimate the LFP as predicted by Eq. (23) and is shown in blue in the bottom plot (blue trace). This predicted LFP can be then compared with the real LFP experimentally recorded (black trace).

Qualitative comparison between the estimated and real LFP signals suggest strong similarities. The time to peak, polarity, and the relative amplitude of peaks are comparable though 

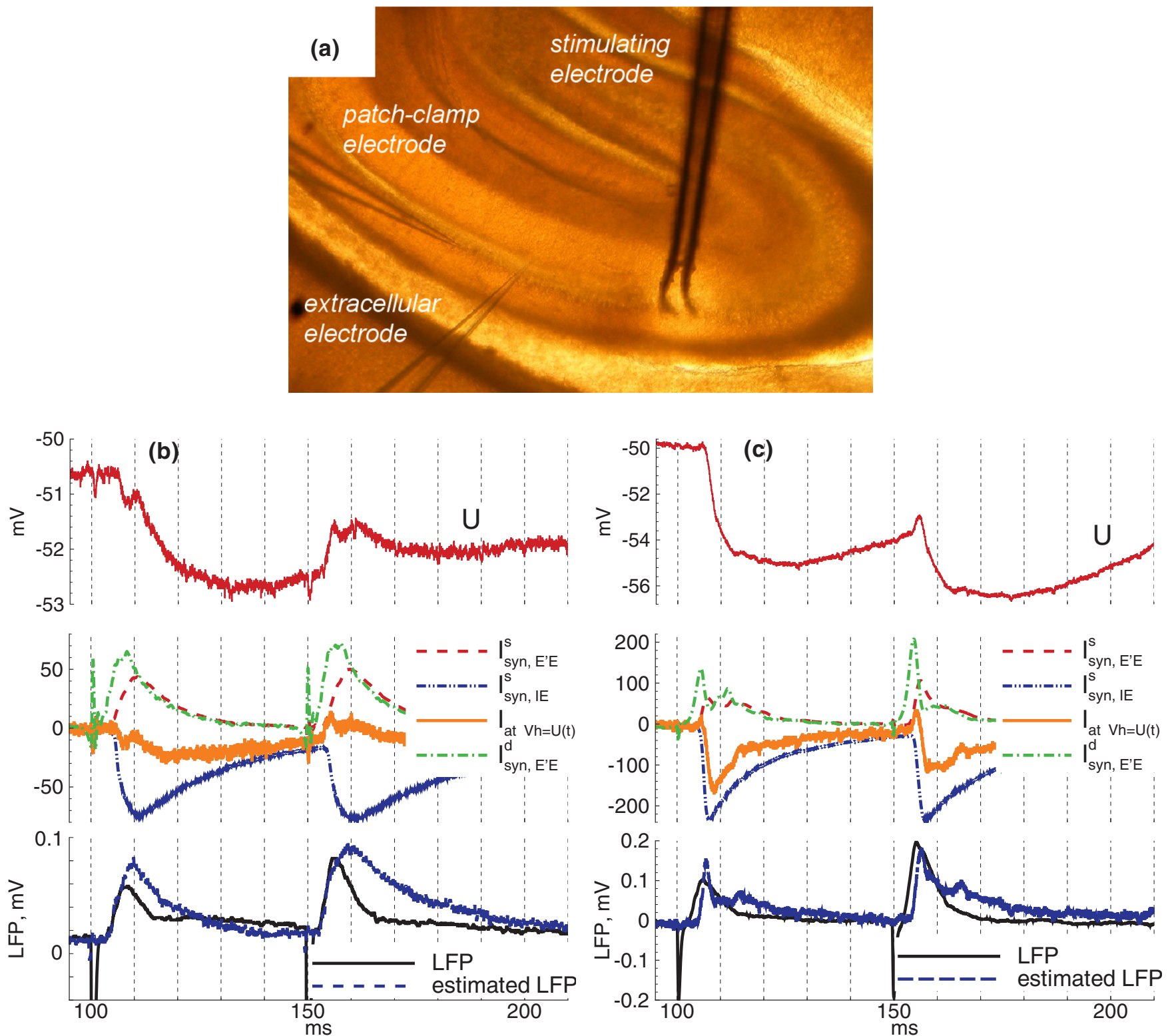

FIG. 2. (Color online) Experimental validation. (a) Image of the experimental preparation. Hippocampal slices were used to validate LFP estimations from Eq. (12). These experiments were part of ongoing projects in the laboratory [40]. A bipolar stimulating electrode (right) was placed to activate the CA2 region of the hippocampus which inputs into the dendrites of CA1 pyramidal cells. Individual pyramidal cells were recorded with patch pipettes filled with cesium-gluconate $(40 \mathrm{mM})$ and K-gluconate $(90 \mathrm{mM})$ solutions while a second glass pippette filled with extracellular solution was used to record LFP from the stratum pyramidale. (b), Data obtained from one CA1 pyramidal cell (cell 1). Panels from top to bottom show: (top plot) the somatic postsynaptic potential $V$ (red solid line) recorded in current-clamp mode; (middle plot) the somatically measured but dendritically originated excitatory current $I_{\operatorname{syn}, E^{\prime} E}^{s}$ (middle, red dashed line), the somatic inhibitory current $I_{\text {syn, } I E}^{s}$ (blue dash-double-dotted line), the somatic capacity and leak current $I_{C}^{s}+I_{L}^{s} \approx I_{\text {at }} V_{h}=U(t)$ (orange solid line) recorded in dynamic VC mode, and the dendritic synaptic current $I_{\mathrm{syn}, E^{\prime} E}^{d}$ (green dash-dotted line) estimated by Eq. (22) from $I_{\mathrm{syn}, E^{\prime} E}^{s}$; (bottom plot) the estimated LFP according to Eq. (23) (blue dash line) and the recorded LFP (black solid line) are shown at the bottom plot. (c) Same as in (b) for another CA1 pyramidal cell (cell 2). The passive parameters of input conductance $G_{L}$ and membrane time constant $\tau_{m}$ were estimated to be $6.5 \mathrm{nS}$ and $15 \mathrm{~ms}$ for cell 1 and $13 \mathrm{nS}$ and $12 \mathrm{~ms}$ for cell 2; $V_{\text {rest }}$ was about $-50 \mathrm{mV}$ for both cells. The dendritic synaptic current $I_{\text {syn, } E^{\prime} E}$ was estimated by Eq. (22) from $I_{\mathrm{syn}, E^{\prime} E}^{s}$ with $l=0.5 \underline{[41]}$ and corresponding $\tau_{m}$.

not identical. Quantitative differences between these signals would suggest deviation of decay time and some early fast components. Most of this residual contribution may result from the fact that the LFP signal, by its nature, reflects averaged activity across a cellular population, whereas the estimate in Eq. (23) is more likely to reflect the contribution of a single cell to that population LFP. This can be further exploited experimentally to estimate the partial contribution of different cell types to the global LFP signal [40]. Importantly, we noted that TCN model estimation of LFP signals resulting from active excitatory and inhibitory currents flowing into the dendrite and soma were roughly similar to estimations obtained from 
two-population models of excitatory and inhibitory neurons (see Appendix A).

\section{B. Experiment 2}

Most experimental data are obtained using the VC mode without dynamic control. We therefore also checked for the ability of our model to predict extracellular LFP signals by Eq. (11) using direct estimations of synaptic currents. To this purpose, as in experiment 1, the compound synaptic current resulting from dendritic activation and feedforward perisomatic inhibition was measured at the soma under VC mode at different holding voltage levels. From these recordings the excitatory and inhibitory conductances $g_{E^{\prime} E}$ and $g_{I E}$ were estimated by the method from Ref. [36], as well as the resting voltage $V_{\text {rest }}$ and the input conductance at rest $G_{i n}$. Then the conductances were set as an input to a neuron governed by Eqs. (20)-(22) with $\tau_{m}^{0}$ and $G_{i n}$ measured from $\mathrm{CC}$ recordings with conductance-step stimulation, and the parameters $l$ and $\gamma$ fitted to match the modeled and recorded LFPs for one of the cells. The examples of the recorded and estimated LFPs are presented in Fig. 3(a). The shape of the
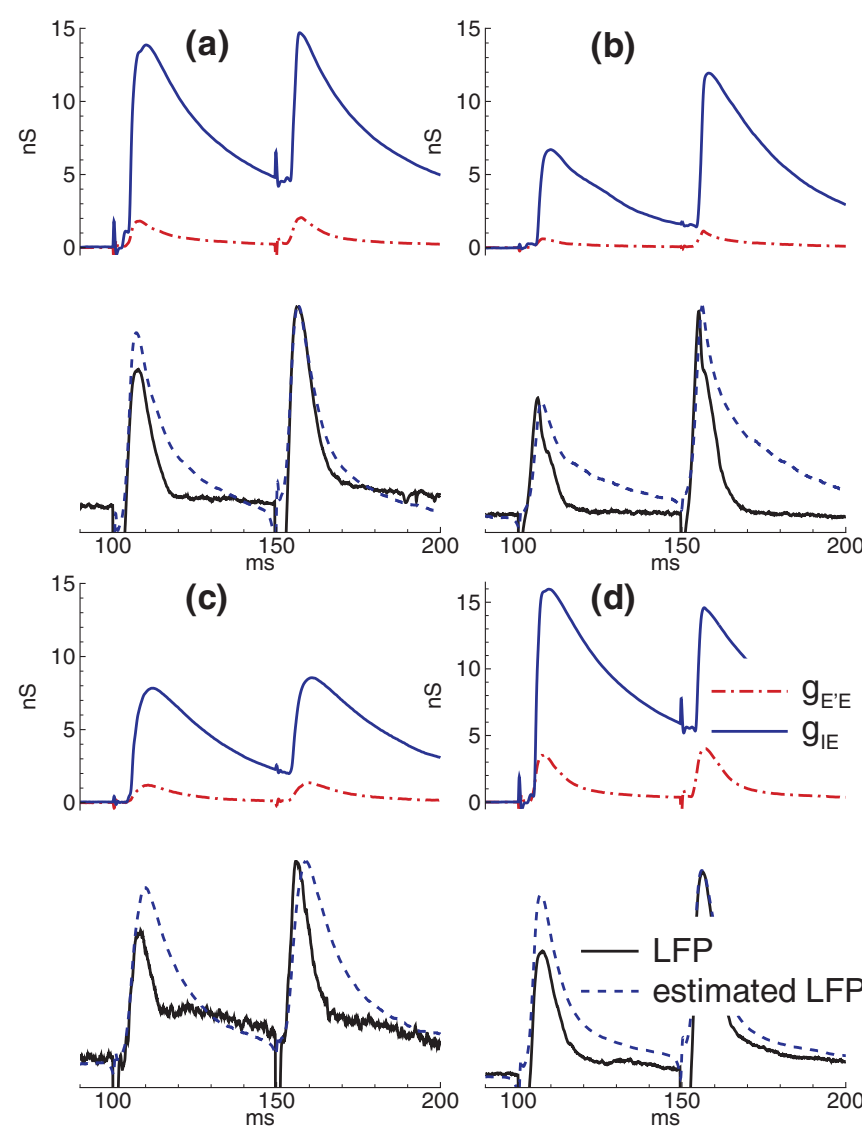

FIG. 3. (Color online) Experimental validation. Excitatory (dash-dotted red lines) and inhibitory (solid blue lines) conductances estimated from voltage-clamp recordings in four cells (a)-(d). LFPsignals registered simultaneously with the intracellular recordings (solid black lines) and estimated by Eqs. (11) and (20)-(22) (dashed blue lines), using the estimated conductances and the parameters $V_{E}=0, V_{I}=-65 \mathrm{mV}, G_{\text {in }}=10 \mathrm{nS}, \tau_{m}=15 \mathrm{~ms}$, $l=4, \gamma=1$. signals, their polarity, the timing of the peaks, and the order of magnitude are qualitatively similar for the model and the experiments, as in experiment 1.

We also compared the proposed LFP model with the models mentioned in the Introduction that represent the LFP as a signal proportional to $\mid$ EPSC $|+|$ IPCS $\mid$ (i.e., $\left|I_{\text {syn, } E^{\prime} E}^{S}\right|+\left|I_{\text {syn }, I E}^{S}\right|$ ) or a signal proportional to the somatic voltage $U$. We computed the coefficients of correlation between the real LFP and each of the signals reconstructed with the three models on a base of the estimated synaptic conductances and found out with the $t$ test (eight trials in six cells) that with $99 \%$ confidence our model provides the highest correlation $(0.86)$.

We conclude that in comparison to the phenomenologically derived models of LFP for one-compartment models mentioned in the Introduction, the proposed simplified twocompartment model is supported by its analytic derivation from the cable equation and the Poisson equation, as well as by experiments thus providing the simplest biologically inspired formulation of LFP in layered neural tissue.

\section{MODELING LFP FROM A SINGLE POPULATION OF INTEGRATE-AND-FIRE TWO-COMPARTMENT NEURONS}

Finally, having proved the validity of the single-neuron model we aimed to generalize our formalism to the population level by considering synaptic activity emerging from a population of discharging cells. To this purpose, we generalized the firing-rate- (FR) type model of a single population previously proposed in Ref. [42] to the case of two-compartment leaky integrate-and-fire (LIF) neurons. The model consists of the Eqs. (20) and (21) and the following formulas for the firing rate:

$$
\begin{gathered}
v(t)=A\left(U, g_{\text {syn }}\right)+B(U, d U / d t) \\
A\left(U, g_{\text {syn }}\right) \\
=\left\{\frac{\sqrt{\pi} \tau_{m}^{0}}{1+g_{\text {syn }} / g_{L}} \int_{\left(V_{\text {reset }}-U\right) / \sigma_{V} \sqrt{2}}^{\left(V_{\mathrm{th}}-U\right) / \sigma_{V} \sqrt{2}} e^{u^{2}}[1+\operatorname{erf}(u)] d u\right\}^{-1} \\
\approx \frac{1+g_{\text {syn }} / g_{L}}{\tau_{m}^{0} V_{\mathrm{th}}}\left[U-V_{\mathrm{th}}-2 \sigma_{V}\right]_{+}, \\
B(U, d U / d t)=\frac{1}{\sqrt{2 \pi} \sigma_{V}}\left[\frac{d U}{d t}\right]_{+} \exp \left[-\frac{\left(U-V_{\mathrm{th}}\right)^{2}}{2 \sigma_{V}^{2}}\right]
\end{gathered}
$$

where steady and nonsteady regimes of firing are considered separately and described by the terms $A$ and $B$, respectively. The formula (25) for the steady-state regime was derived in [43] for one-compartment LIF neurons; its approximation is empiric, where $g_{\text {syn }}$ is the total synaptic conductance; $\tau_{m}=C /\left(g_{L}+g_{\text {syn }}\right)$. The formula (26) for $B$ was derived by considering variability of membrane voltage between neurons according to Gaussian distribution around the mean, $U$. If the mean voltage $U(t)$ is monotonically increasing, then the mean firing rate can be calculated from the distribution provided a constant threshold value, $V_{\text {th }}$.

Comparison of such a firing-rate model of a population of one-compartment LIF neurons with a refractory-density model was presented in Ref. [42] and for adaptive neurons in Ref. [30]. The comparison revealed that the FR model correctly 
reproduces steady states [i.e., the term $A$ in Eq. (24)] as well as population rate bursts in response to rapid voltage rise during strong stimulation [i.e., the term $B$ in Eq. (24)].

The value of $U$ and $d U / d t$ in the firing-rate model based on Eqs. (24)-(26) can be estimated from the two-compartment model for the subthreshold voltage based on Eqs. (20)-(22). In order to take into account the contribution of spikes into the LFP, we introduce a phenomenological extention to Eq. (12). To this purpose, we assume that the contribution of sodium channels is proportional to the charge of sodium ions transferred during a single spike multiplied by the spike frequency. The sodium current profile during a spike is a triangle-like function, thus the charge is approximated as $I_{\mathrm{Na}}^{\max } \tau^{\mathrm{sp}} / 2$, where $I_{\mathrm{Na}}^{\max }$ is the sodium channel amplitude and $\tau^{\mathrm{sp}}$ is the duration of a spike. The sodium current contributes into $J_{m}^{s}$, resulting in the following generalized formula for the LFP:

$\varphi(t)=\frac{p L}{2 \sigma r_{i}}\left\{\left[C \frac{d U}{d t}+g_{L} U-\frac{\tau^{\mathrm{sp}} I_{N a}^{\max }}{2} v(t)\right] S^{\mathrm{soma}}-I_{\text {syn }}^{s}\right\}$.

Consequently, the simplest mean-field model relating synaptic currents to multiunit activity and LFP consists of Eqs. (20)-(22) for somatic voltage, Eqs. (24)-(26) for the firing rate, and Eqs. (27) for the LFP.

\section{SUMMARY}

In summary, we have derived the simplest analytical estimation of synaptic LFP signals [Eq. (11) or Eq. (12)] resulting from single neurons, based on a two-compartment model that account for simple somatodendritic integration of synaptic inputs in layered structures. This model is supported by experimental validation using extracellular stimulation of the layered input pathway and patch recordings in hippocampal slices. It is also consistent with the phenomenological consideration of LFP as the difference of dendritic and somatic membrane voltages [23] and the analytical solution obtained by Ref. [32] for a particular case of homogeneously distributed cylinders with a certain synaptic input current.

We further extend our model [Eq. (27)] to account for the contribution of synaptic activity resulting from multiunit firing in a simple integrate-and-fire analytical model of twocompartment neurons. Together, these models provide the simplest biologically inspired formulation of LFP in layered neural tissue and can be further exploited to estimate the contribution of different neuronal types to population LFP dynamics.

\section{ACKNOWLEDGMENTS}

This work was supported by Grants No. 13-04-01835a, No. 15-29-01344-ofi-m, and No. 15-04-06234a from the Russian Foundation for Basic Research to A.C. and BFU201237156-C03-01 from the Spanish Ministerio de Economía y Competitividad to L.M.P.

\section{APPENDIX: LFP IN MEAN-FIELD MODELS OF RECURRENTLY CONNECTED POPULATIONS}

In order to clarify the contribution of different membrane currents to the evoked LFP signal, we model recurrently connected populations, based on Eqs. (12)-(14) and (23) (26) for excitatory $(E)$ and inhibitory $(I)$ neurons, both excited by incoming collaterals $\left(E^{\prime}\right)$. For simplicity, we assume that excitatory and inhibitory neurons are the same in biophysical terms. The inhibitory synapses are located on soma, thus the inhibitory synaptic currents on somas of excitatory and inhibitory neurons are $I_{\mathrm{syn}, I E}^{s}(t)=$ $g_{I E}(t)\left[V^{E}(t)-V_{I}\right]$ and $I_{\text {syn }, I I}^{s}(t)=g_{I I}(t)\left[V^{I}(t)-V_{I}\right]$, correspondingly, where double subscripts $I E$ and $I I$ mark the types of pre- and postsynaptic neurons, $V_{I}$ is the reversal potential, and $g_{I E}(t)$ and $g_{I I}(t)$ are somatically measured synaptic conductances. The excitatory synapses are on the dendrites, thus they are estimated according to (14) as follows: $I_{\text {syn }, E^{\prime} E}^{d}(t)=\left(l / 2 \tau_{m}^{0} d / d t+1+l / 2\right) I_{\text {syn, } E^{\prime} E}^{s}(t)$ and $I_{\text {syn, } E^{\prime} I}^{d}(t)=\left(l / 2 \tau_{m}^{0} d / d t+1+l / 2\right) I_{\text {syn, } E^{\prime} I}^{s}(t)$, where somatically measured currents are approximated as $I_{\text {syn, } E^{\prime} E}^{s}(t)=$ $g_{E^{\prime} E}(t)\left[V^{E}(t)-V_{E}\right], I_{\text {syn, } E^{\prime} I}^{s}(t)=g_{E^{\prime} I}(t)\left[V^{I}(t)-V_{E}\right] ; V_{E}$ is the reversal potential of excitatory currents. The synaptic kinetics is determined by the following equations expressed via synaptic gating variables $m_{I}(t)$ and $m_{E^{\prime}}(t)$ controlled by presynaptic rates of inhibitory neurons $v_{I}(t)$ and incoming collaterales $v_{E^{\prime}}(t)$ :

$$
\begin{gathered}
g_{I E}(t)=\bar{g}_{I E} m_{I}(t), g_{I I}(t)=\bar{g}_{I I} m_{I}(t), \\
g_{E^{\prime} E}(t)=\bar{g}_{E^{\prime} E} m_{E^{\prime}}(t), g_{E^{\prime} I}(t)=\bar{g}_{E^{\prime} I} m_{E^{\prime}}(t), \\
\tau_{I}^{2} \frac{d^{2} m_{I}}{d t^{2}}+2 \tau_{I} \frac{d m_{I}}{d t}+m_{I}=e \tau_{I} v_{I}(t), \\
\tau_{E}^{2} \frac{d^{2} m_{E^{\prime}}}{d t^{2}}+2 \tau_{E} \frac{d m_{E^{\prime}}}{d t}+m_{E^{\prime}}=e \tau_{E} v_{E^{\prime}}(t) .
\end{gathered}
$$

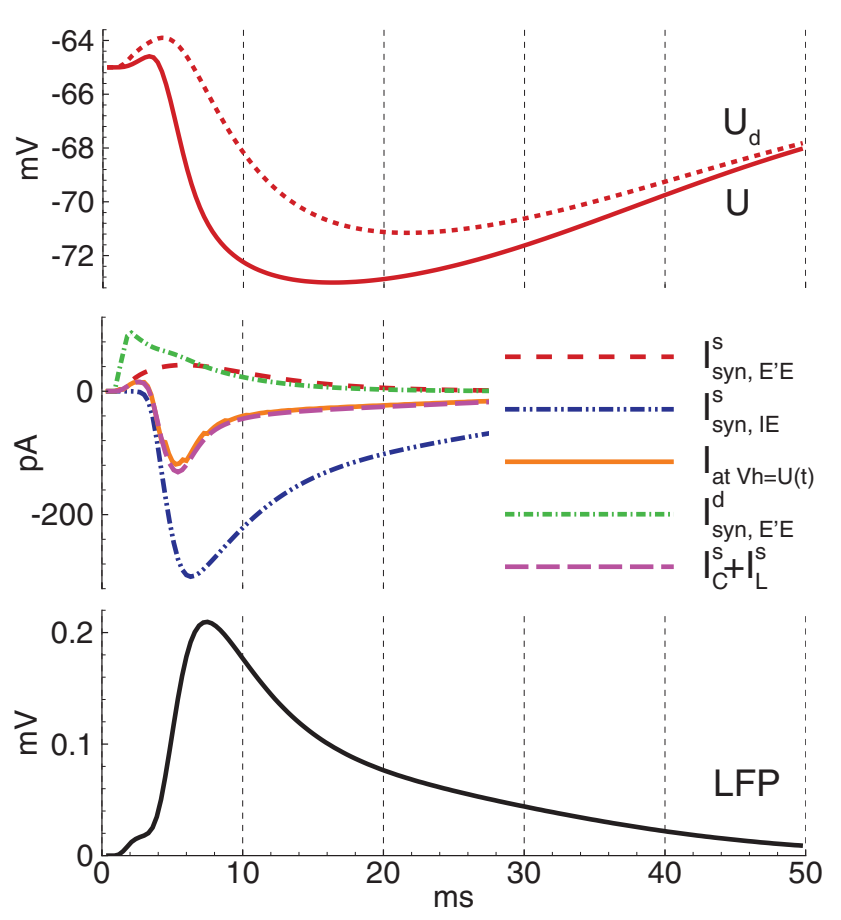

FIG. 4. (Color online) Simulation of recurrently connected populations evoked by a pulse of activity of the incoming collaterales with two-population mean-field model. 
For the case of electric stimulation of the incoming collaterales, the firing rate $v_{E^{\prime}}(t)$ is set as a $\delta$ function. The constant parameters $\bar{g}_{E^{\prime} E}, \bar{g}_{E^{\prime} I}, \bar{g}_{I E}$, and $\bar{g}_{I I}$ are the maximum synaptic conductances; $\tau_{E}, \tau_{I}$ are the synaptic kinetics time constants. For simulation the following parameter values were chosen: $\bar{g}_{E^{\prime} E}=2.5 \mathrm{nS}, \bar{g}_{E^{\prime} I}=25 \mathrm{nS}, \bar{g}_{I E}=\bar{g}_{I I}=250 \mathrm{nS}, \tau_{E}=4$ $\mathrm{ms}, \tau_{I}=7 \mathrm{~ms} ; V_{\mathrm{th}}-V_{L}=8 \mathrm{mV}, V_{\text {reset }}=V_{L}, \sigma_{V}=2 \mathrm{mV}$, $\tau_{m}^{0}=15 \mathrm{~ms}, G^{s}=3.3 \mathrm{nS}, \gamma=3, l=0.5$.
The simulation presents the currents in excitatory neurons, which contribute to the LFP $\varphi(t)$ (Fig. 4). The membrane potential, LFP and synaptic currents are similar to the experimental signals shown in Fig. 2. Also note that the current $I_{\text {at }} V_{h}=U(t)$, obtained as if in voltageclamp mode with the holding voltage equal to $V^{E}(t)$, is close to $I_{C}^{S}+I_{L}^{S}$, i.e., it might be estimated by somatic measurements.
[1] G. Buzsaki, C. A. Anastassiou, and C. Koch, The origin of extracellular fields and currents-EEG, ECoG, LFP and spikes, Nat. Rev. Neurosci. 13, 407 (2012).

[2] C. Bedard, H. Kroger, and A. Destexhe, Model of low-pass filtering of local field potentials in brain tissue, Phys. Rev. E 73, 051911 (2006).

[3] C. Bedard and A. Destexhe, Generalized theory for currentsource-density analysis in brain tissue, Phys. Rev. E 84, 041909 (2011).

[4] C. Bedard and A. Destexhe, Generalized cable theory for neurons in complex and heterogeneous media, Phys. Rev. E 88, 022709 (2013).

[5] C. Bedard and A. Destexhe, Generalized cable formalism to calculate the magnetic field of single neurons and neuronal populations, Phys. Rev. E 90, 042723 (2014).

[6] C. Alvarado-Rojas, G. Huberfeld, M. Baulac, S. Clemenceau, S. Charpier, R. Miles, L. M. de la Prida, and M. Le Van Quyen, Different mechanisms of ripple-like oscillations in the human epileptic subiculum, Ann. Neurol. 77, 281 (2015).

[7] J. B. Ranck, Jr. and S. L. BeMent, The specific impedance of the dorsal columns of cat: An anisotropic medium, Exp. Neurol. 11, 451 (1965).

[8] C. Nicholson, Theoretical analysis of field potentials in anisotropic ensembles of neuronal elements, IEEE Trans. Biomed. Eng. BME-20, 278 (1973).

[9] C. Nicholson and J. A. Freeman, Theory of current sourcedensity analysis and determination of conductivity tensor for anuran cerebellum, J. Neurophysiol. 38, 356 (1975).

[10] N. K. Logothetis, C. Kayser, and A. Oeltermann, In vivo measurement of cortical impedance spectrum in monkeys: Implications for signal propagation, Neuron 55, 809 (2007).

[11] H. Linden, T. Tetzlaff, T. C. Potjans, K. H. Pettersen, S. Grun, M. Diesmann, and G. T. Einevoll, Modeling the spatial reach of the LFP, Neuron 72, 859 (2011).

[12] E. W. Schomburg, C. A. Anastassiou, G. Buzsaki, and C. Koch, The spiking component of oscillatory extracellular potentials in the rat hippocampus, J. Neurosci. 32, 11798 (2012).

[13] C. Gold, D. A. Henze, C. Koch, and G. Buzsaki, On the origin of the extracellular action potential waveform: A modeling study, J. Neurophysiol. 95, 3113 (2006).

[14] G. T. Einevoll, C. Kayser, N. K. Logothetis, and S. Panzeri, Modelling and analysis of local field potentials for studying the function of cortical circuits, Nat. Rev. Neurosci. 14, 770 (2013).

[15] J. M. Ibarz, G. Foffani, E. Cid, M. Inostroza, and L. M. de la Prida, Emergent dynamics of fast ripples in the epileptic hippocampus, J. Neurosci. 30, 16249 (2010).

[16] A. V. Chizhov, Conductance-based refractory density model of primary visual cortex, J. Comp. Neurosci. 36, 297 (2014).
[17] A. V. Chizhov and L. J. Graham, Efficient evaluation of neuron populations receiving colored-noise current based on a refractory density method, Phys. Rev. E 77, 011910 (2008).

[18] J. Baladron, D. Fasoli, O. Faugeras, and J. Touboul, Mean-field description and propagation of chaos in networks of HodgkinHuxley and FitzHugh-Nagumo neurons, J. Math. Neurosci. 2, 10 (2012).

[19] P. L. Nunez, Electric Fields of the Brain: The Neurophysics of EEG (Oxford University Press, Oxford, 1981).

[20] A. Mazzoni, S. Panzeri, N. K. Logothetis, and N. Brunel, Encoding of naturalistic stimuli by local field potential spectra in networks of excitatory and inhibitory neurons, PLoS Comp. Biol. 4, e1000239 (2008).

[21] A. Destexhe, Spike-and-wave oscillations based on the properties of GABAB receptors, J. Neurosci. 18, 9099 (1998).

[22] A. Destexhe and C. Bedard, Local field potential, Scholarpedia 8, 10713 (2013).

[23] S. Demont-Guignard, P. Benquet, U. Gerber, and F. Wendling, Analysis of intracerebral EEG recordings of epileptic spikes: Insights from a neural network model, IEEE Trans. Biomed. Eng. 56, 2782 (2009).

[24] P. Beim Graben and S. Rodrigues, A biophysical observation model for field potentials of networks of leaky integrate-and-fire neurons, Front. Comput. Neurosci. 6, 100 (2012).

[25] A. Destexhe and C. Bedard, Do neurons generate monopolar current sources? J. Neurophysiol. 108, 953 (2012).

[26] W. Rall and G. M. Shepherd, Theoretical reconstruction of field potentials and dendrodendritic synaptic interactions in olfactory bulb, J. Neurophysiol. 31, 884 (1968).

[27] A. N. Pokrovskii, A reverse problem of cortical extracellular potential modeling, Sist. Upravl. Inf. Tekhnol. 1, 94 (2006).

[28] F. Barbieri, A. Mazzoni, N. K. Logothetis, S. Panzeri, and N. Brunel, Stimulus dependence of local field potential spectra: Experiment versus theory, J. Neurosci. 34, 14589 (2014).

[29] A. V. Chizhov, A two-compartment model for the dependence of a postsynaptic potential on a postsynaptic current, measured by the patch-clamp method, Biofizika 49, 877 (2004).

[30] A. Ju. Buchin and A. V. Chizhov, Modified firing-rate model reproduces synchronization of a neuronal population receiving complex input, Optical Memory and Neural Networks (Information Optics) 19, 166 (2010).

[31] A. V. Chizhov and L. J. Graham, Population model of hippocampal pyramidal neurons, linking a refractory density approach to conductance-based neurons, Phys. Rev. E 75, 011924 (2007).

[32] C. Nicholson and R. Llinas, Field potentials in the alligator cerebellum and theory of their relationship to Purkinje cell dendritic spikes, J. Neurophysiol. 34, 509 (1971). 
[33] D. J. Griffiths, Introduction to Electrodynamics, 3rd ed. (Prentice Hall, Upper Saddle River, NJ, 2007).

[34] A. R. Sargsyan, C. Papatheodoropoulos, and G. K. Kostopoulos, Modeling of evoked field potentials in hippocampal CA1 area describes their dependence on NMDA and GABA receptors, J. Neurosci. Methods 104, 143 (2001).

[35] P. F. Pinsky and J. Rinzel, Intrinsic and network rhythmogenesis in a reduced Traub model for CA3 neurons, J. Comput. Neurosci. 1, 39 (1994).

[36] L. J. Borg-Graham, C. Monier, and Y. Fregnac, Visual input evokes transient and strong shunting inhibition in visual cortical neurons, Nature 393, 369 (1998).

[37] S. A. Fazeli, A. M. Gharravi, M. Jahanshahi, S. Ghafari, N. Behnampour, and M. J. Golalipour, Resistance of CA1 pyramidal cells to STZ-induced diabetes in young rats, Int. J. Morphol. 27, 997 (2009).

[38] G. Holt, A Critical Reexamination of some assumptions: Implications of cable theory in neurobiology, Ph.D. thesis, California Institute of Technology, 1998.
[39] F. Laurent, J. R. Brotons-Mas, E. Cid, D. Lopez-Pigozzi, M. Valero, B. Gal, and L. M. de la Prida, Proximodistal structure of theta coordination in the dorsal hippocampus of epileptic rats, J. Neurosci. 35, 4760 (2015).

[40] M. Valero, E. Cid, R. G. Averkin, J. Aguilar, A. SanchezAguilera, T. J. Viney, D. Gomez-Dominguez, E. Bellistri, L. M. de la Prida, Determinants of different deep and superficial CA1 pyramidal cell dynamics during sharp-wave ripples, Nat. Neurosci. 18, 1281 (2015).

[41] D. Turner and P. Schwartzkroin, Electrical characteristics of dendrites and dendritic spines in intracellularly stained CA3 and dentate hippocampal neurons, J. Neurosci. 3, 2381 (1983).

[42] A. V. Chizhov, S. Rodrigues, and J. Terry, A comparative analysis of a firing-rate model and a conductance-based neural population model, Phys. Lett. A 369, 31 (2007).

[43] P. I. M. Johannesma, Diffusion models of the stochastic acticity of neurons, Neural Networks (Springer, Berlin, 1968), pp. 116-144. 